\title{
Resumen de Actividades del Comité de Tuberculosis. Región Metropolitana, 2007. Selección de Temas
}

\author{
Activities of the Tuberculosis Committee of Santiago during 2007. \\ A summary of selected sessions
}

En Santiago de Chile ha estado funcionando, en forma ininterrumpida, desde hace más de 40 años, un comité que asesora al Programa Nacional de Control de la Tuberculosis del Ministerio de Salud. Esta debe ser una de las actividades médicas especializadas con mayor continuidad, a nivel de un país en desarrollo.

El Comité Metropolitano de Tuberculosis se reúne regularmente una vez al mes. De las 10 presentaciones realizadas durante el año 2007, hemos seleccionado 4 de las más representativas.

\section{Resultados del retratamiento de la tuberculosis}

Dr. Carlos Peña M., Coordinador Comité Metropolitano de Tuberculosis.

Zulema Torres, EU, Subdepartamento Prioridades Sanitarias. SEREMI de Salud, Región Metropolitana.

Los enfermos tuberculosos que recaen o los abandonos rescatados (pacientes que han recibido fármacos antituberculosos anteriormente), inician una terapia normada que consiste en un refuerzo con estreptomicina en la fase diaria inicial y de etambutol en la fase intermitente, que se prolonga hasta los 9 meses.

La última evaluación de los resultados del tratamiento de una cohorte de casos de tuberculosis antes tratados (AT), es decir el conjunto de recaídas y abandonos, corresponde al período 1999-2001 (Tabla 1.1). Sobre un total de 469 pacientes, de los cuales cerca del $70 \%$ eran recaídas, se comunicó un $71 \%$ de eficiencia o éxito terapéutico como promedio (alta del paciente con confirmación de negativización bacteriológica), con un porcentaje de abandonos que osciló entre un 14\% (2001) y 22,3\% (1999). Esta información se obtuvo después de un seguimiento riguroso de los enfermos, excluyendo los casos tratados con esquemas terapéuticos diferentes a los vigentes en las normas nacionales en el momento de la evaluación. Los servicios de salud que colaboraron variaron entre 21 (2000) y 28 (2001).

$\mathrm{Al}$ efectuar un consolidado con todas las evaluaciones previas disponibles de retratamientos, se pudo obtener información de 1812 casos entre 1993 y 2001, con un 70,1\% de éxito de tratamiento. Esta eficiencia terapéutica se encuentra reducida debido fundamentalmente al alto porcentaje de abandonos (20\%) y no a una elevada letalidad (sólo 6\% de fallecidos registrados). Los fracasos al retratamiento fueron excepcionales ( 3 casos durante 9 años, 0,2\%).

Para continuar con las evaluaciones de la eficiencia terapéutica en los pacientes AT se revisó la información disponible entre el 2003 y 2005 (Tabla 2.1), aunque sólo pudo obtenerse datos de algunos servicios de salud.

En 100 pacientes evaluados, la eficiencia del tratamiento alcanzó a un promedio de $80 \%$, el porcentaje de abandono se redujo a cerca de un $10 \%$, pero se observó un incremento de la letalidad a un $10 \%$.

Tabla 1.1. Evaluación de una cohorte de pacientes con tuberculosis pulmonar antes tratada.

Chile. 1999-2001

\begin{tabular}{lrrc}
\hline & $\mathbf{1 9 9 9}$ & $\mathbf{2 0 0 0}$ & $\mathbf{2 0 0 1}$ \\
\hline $\mathrm{N}^{\text {o }}$ servicios de salud & 25 & 21 & 28 \\
$\mathrm{~N}^{\circ}$ casos antes tratados & 229 & 154 & 178 \\
\% excluidos & 32 & 34 & 26 \\
\% de Recaídas & 54 & 75 & 79 \\
Resultados del retratamiento (\%): & & \\
- Fallecidos & 5,7 & 9,7 & 10,1 \\
- Abandonos & 22,3 & 18,8 & 14 \\
- Alta (inactivos) & 69,9 & 68,8 & 73 \\
\end{tabular}


Tabla 2.1. Evaluación de una cohorte de 100 pacientes con tuberculosis pulmonar antes tratada. Chile. 2003-2005

\begin{tabular}{lr}
\hline $\mathrm{N}^{\circ}$ casos (a+b) & 100 \\
a) $\mathrm{N}^{\circ}$ de abandonos & 10 \\
b) $\mathrm{N}^{\circ}$ de recaídas & 90 \\
Resultados del retratamiento (\%): & \\
- Alta (inactivo) & 80 \\
- Abandonos & 10 \\
- Fallecidos & 10 \\
\hline
\end{tabular}

Al considerar sólo los casos con abandono previo, la eficiencia se redujo casi en un $40 \%$, ya que en este grupo existe una mayor letalidad (puede alcanzar hasta un 30\%) y una mayor tendencia a repetir el abandono (puede llegar hasta un 20\%). En cambio, en los con antecedentes de recaída, sin abandono previo, la letalidad fue menor $(10 \%)$ y el porcentaje de abandono también $(8,7 \%)$.

La conclusión más importante de estos estudios es que se debe mejorar la eficiencia del tratamiento de la tuberculosis en los pacientes AT y mejorar las estrategias de intervención para pacientes con abandonos previos, ya que tienden a abandonar nuevamente la terapia y tienen mayor riesgo de morir.

\section{El sanatorio de tuberculosis "Complejo Elba Garategua" en la actualidad}

\subsection{Informe del Sanatorio}

\section{Dras. Angélica Ojeda, Judith Álvarez y María Ivánovic. Evelyn Honorato, EU. Sanatorio "Elba Garategua". Servicio de Salud Metropo- litano Sur-Oriente (SSMSO).}

El actual Sanatorio de Tuberculosis de la Región Metropolitana se encuentra en San José de Maipo, una zona precordillerana dependiente del SSMSO. Durante muchos años este sanatorio estuvo dedicado a la hospitalización de enfermos tuberculosos, pero actualmente también atiende otras patologías. Al sanatorio ingresan los enfermos a través de una solicitud de admisión que identifica al paciente y su estado clínico. Este documento es de gran importancia para el manejo de los casos.

Para conocer más a fondo la contribución actual del sanatorio en el manejo de los enfermos de tuberculosis se solicitó al equipo a car- go de los pacientes, la información correspondiente al año 2006. Durante ese año ingresaron 89 enfermos, de los cuales 75 eran varones (84\%). El promedio de edad fue de 46 años, pero casi un $24 \%$ registraba más de 60 años. El promedio de estadía se estableció en 108 días. La procedencia consignaba que un $37 \%$ pertenecía al SSMSO, un $20 \%$ al SSM Centro, un $10 \%$ al SSM Occidente, un 9\% del SSM Norte, un $9 \%$ del SSM Sur y el resto de Servicios de Salud de otras regiones del país.

Sólo un $44 \%$ de los pacientes permanecieron hospitalizados hasta completar el tratamiento. Un $6 \%$ falleció durante la hospitalización, principalmente por complicaciones de la infección con VIH. Destacamos que un $36 \%$ abandonó el tratamiento antes de ser dados de alta, $26 \%$ por fuga desde el recinto y $10 \%$ por mala conducta, que motivó un alta disciplinaria. Otros enfermos solicitaron el traslado a sus consultorios motivados, como en el caso de las mujeres, por la necesidad de atender situaciones familiares, o en los varones por la necesidad de insertarse en actividades laborales.

Uno de los grandes problemas en el manejo de estos pacientes es la asociación con alcoholismo (casi en un 50\%) o drogadicción. También son relevantes las comorbilidades en las edades mayores, la asociación con VIH/SIDA $(12,3 \%)$, desnutrición (21\%) y trastornos psiquiátricos $(11,2 \%)$. Las reacciones adversas a medicamentos antituberculosos durante el tratamiento fueron frecuentes $(13,4 \%)$, siendo en la mitad de los casos debidos a toxicidad hepática.

\subsection{Hacia un mejor manejo de la tuberculosis en el Sanatorio "Elba Garategua"}

Drs. Carlos Peña M. Equipo Técnico de Tuberculosis. SSM Centro.

Alvaro Yáñez. Equipo Técnico de TBC. SSM Occidente.

Patricio Olivares y Zulema Torres, EU. Subdepartamento Prioridades Sanitarias. SEREMI de Salud, Región Metropolitana.

La lucha contra la tuberculosis en Chile nos ha permitido alcanzar la menor incidencia histórica de la enfermedad, que ahora se va concentrando en grupos vulnerables. Pero estos grupos muestran una menor eficiencia terapéutica, principalmente por el riesgo de abandonar la terapia.

La participación de un sanatorio en la lucha contra la tuberculosis en el momento actual es todavía importante, no sólo por permitir una 
asistencia médica más directa, sino para asegurar un mejor control del tratamiento (supervisión, seguimiento bacteriológico y tolerancia a los medicamentos), reducir el riesgo de contagio comunitario al sustraer al paciente de su medio, permitir el manejo de las patologías asociadas, apoyar la recuperación de la desnutrición y de hábitos nocivos, especialmente alcoholismo, y establecer lazos afectivos con pacientes socialmente aislados.

El sistema de manejo hospitalario debe asegurar un tratamiento bajo estándares de DOTS (tratamiento abreviado directamente supervisado), evitando la aparición de fracasos y multirresistencia, controlando las reacciones adversas a drogas precozmente y preparando al paciente para su reinserción social.

Para que esto pueda ser posible, es necesario introducir mejorías en algunos aspectos operacionales y de gestión a nivel del sanatorio, según se desprendió de una visita de terreno y del intercambio de información con el equipo de salud involucrado en el manejo de los casos.

Entre las medidas propuestas se mencionan:

1. Establecer un protocolo de ingreso y criterios de exclusión de hospitalización en el sanatorio.

2. Adecuar la información suministrada por la solicitud de admisión.

3. Establecer zonas con aislamiento efectivo para casos contagiosos.

4. Conformar un equipo multidisciplinario de manejo constituido por psicólogo o psiquiatra, asistente social, nutricionista e internista.

5. Establecer una coordinación más estrecha con los equipos técnicos de tuberculosis de los servicios de salud.

6. Mantener una capacitación continua en el manejo de la tuberculosis, para todos los componentes del equipo de salud del sanatorio.

7. Mantener informados mensualmente a los servicios de salud de la región metropolitana sobre el estado de los casos enviados desde estos servicios.

8. Elaborar un instructivo de los derechos y deberes de los pacientes en el sanatorio para dárselos a conocer al ingreso.

9. Implementar programas de laborterapia y actividades sustentables o recreativas para los pacientes con larga estadía.

10.Disponer de transporte de los egresados hacia sus domicilios y lugares donde seguir la terapia.

11. Avisar oportunamente al equipo técnico de tuberculosis del servicio de salud correspondiente, los egresos por cualquier circunstancia.
Estas recomendaciones se acompañan, además, de otras sugerencias como mejoras estructurales, incluyendo refacciones de salas y baños, adecuación de salas para aislamiento y habilitación de talleres para pacientes.

Se sugiere, además, un buen control de los ingresos (visitas) y egresos transitorios (salidas de pacientes). Por último, es de la mayor importancia incrementar o por lo menos mantener el número de horas funcionarias del equipo actual del sanatorio.

\section{Evolución de la tuberculosis en la Región Metropolitana}

Dr. Carlos Peña M. Equipo Técnico de Tuberculosis. SSM Centro.

Zulema Torres. EU, Subdepartamento de prioridades sanitarias. SEREMI de Salud, Región Metropolitana.

\section{La Tuberculosis en la Región Metropolitana. 2005}

El número total de casos fue de 897 pacientes, lo que generó una tasa de 13,6/100.000 habitantes. La Tabla 1.3 muestra la tasa de incidencia de tuberculosis en los diferentes servicios de salud de la Región Metropolitana por separado. La tendencia a la reducción de la tuberculosis en los diversos servicios fluctuó sólo entre $-0,3 \%$ y $-0,7 \%$. El $51 \%$ de los casos eran bacilíferos (tasa de 6,9/100.000), lo que significó una reducción de $-0,9 \%$ con respecto al año anterior. Se determinó que 16 comunas tienen tasas sobre 20/100.000. La letalidad promedio fue de 8,8\%; sin embargo, el SSM Norte, el SSM Centro y el SSM Oriente mostraron una letalidad inferior al 5\%.

Debemos precisar que entre los años 2001 y 2005 la tasa de tuberculosis en la Región Metropolitana se redujo de 18,7 a 14,9/100.000 para todas las localizaciones y para las formas bacilíferas de 9,4 a 6,9/100.000.

Tabla 1.3. Tasa de incidencia de tuberculosis en todas las localizaciones en los Servicios de Salud de la Región Metropolitana. 2005

\begin{tabular}{lc}
\hline Servicio de Salud RM & tasa/100.000 \\
\hline Occidente & 19,1 \\
Sur & 17,6 \\
Norte & 16,8 \\
Centro & 15,3 \\
Sur-Oriente & 8,8 \\
Oriente & 7,1 \\
\hline
\end{tabular}




\section{La Tuberculosis en la Región Metropolitana. 2006}

Mediante la información suministrada por los boletines de enfermedades de notificación obligatoria y el registro nacional de casos, que muestran una concordancia de información de un 95\%, se notificaron 984 casos de tuberculosis en la Región Metropolitana el año 2006, correspondiente a una tasa de 14,9/100.000, similar a la de 2005. La distribución por género mostró que 617 casos corresponden a varones (62\%) con tasa de 19,1/100.000 y 367 casos son mujeres, con tasa de 10,8/100.000. Los casos pulmonares bacilíferos, vírgenes a tratamiento, alcanzaron a 453 (46\% de la incidencia), con una tasa de 6,9/100.000.

Los servicios de salud metropolitanos tienen tasas de tuberculosis en todas sus formas muy variables (Tabla 2.3); en todo caso, en todos -excepto en el SSM Occidente- es inferior a 20/100.000 habitantes.

En relación a la distribución de tasas de incidencia de tuberculosis en las 52 comunas de la región metropolitana (Tabla 2.3), 16 comunas tienen una tasa mayor ó igual a 20/100.000. Se puede decir entonces que el $30 \%$ de las comunas, que albergan al $19 \%$ de la población, se encuentran en situación epidemiológica desfavorable. Al comparar la situación con respecto al 2001, se aprecia una franca mejoría, ya que en el 2001 el 40,3\% de las comunas tenía tasa superior a 20/100.000.

En relación a la distribución de casos por edades, se notificaron 27 casos de tuberculosis entre menores de 15 años. En este grupo etáreo hubo 9 menores de 5 años, pero no hubo casos

Tabla 2.3. Distribución de las tasas de incidencia de la tuberculosis en todas sus formas en los 6 Servicios de Salud y en las 52 comunas de la Región Metropolitana. 2006

\begin{tabular}{llc}
\hline $\begin{array}{l}\text { Tasa/ } \\
\mathbf{1 0 0 . 0 0 0}\end{array}$ & Servicio de Salud & $\begin{array}{c}\text { Número de } \\
\text { comunas }\end{array}$ \\
\hline$<10$ & Oriente y Sur-Oriente & 16 \\
$10-20$ & Centro, Norte y Sur & 20 \\
$\geq 20$ & Occidente & 16 \\
\hline
\end{tabular}

de meningitis. También es importante mencionar que 3 de los menores eran hijos de inmigrantes recientes. El $70 \%$ de los casos se encuentra entre los 15 y 64 años (758 casos) lo que se traduce en una tasa de 16,8/100.000 para ese grupo de edad. Pero se debe destacar especialmente, al grupo de adultos mayores de 65 años, en el cual se diagnosticaron 199 casos, con una tasa de 38/100.000 (Tabla 3.3). Uno de los datos más impresionantes fue la presencia de 50 casos de tuberculosis en mayores de 80 años ( $25 \%$ de la incidencia del adulto mayor).

La intensidad de búsqueda de casos de tuberculosis pulmonar, a través de la baciloscopía de expectoración se ha frenado e incluso ha disminuido en los últimos años (Tabla 4.3). El 2001 se realizaron 123.574 baciloscopías, contrastando con las 116.086 del 2006 (7.488 menos, lo que significa un $6 \%$ de reducción). Sólo por esta disminución, considerando una positividad cercana al 1\%, se habrían dejado de diagnosticar 55 casos.

En relación a los grupos vulnerables, el 33,6\% tenía esta condición (331 casos), siendo la condición de adulto mayor la más frecuente. Un $5,7 \%$ de casos eran $\mathrm{VIH}+$, un $3,6 \%$ tenía la condición de inmigrante, un $3,2 \%$ se encontraba en reclusión (a pesar del bajo número de casos respecto de su población penal, este grupo genera altas tasas de tuberculosis, cercanas a 338/100.000), un 1,2\% estaba en indigencia y un $20 \%$ correspondía al adulto mayor, lo que obliga a focalizar la pesquisa en este grupo de edad, que constituye el $60 \%$ de todos los grupos de riesgo (Tabla 5.3).

Además, se encontró que el $50 \%$ de los ca-

Tabla 3.3. Tasa de incidencia de la tuberculosis en todas sus formas según grupo de edad. Región Metropolitana 2006

\begin{tabular}{lc}
\hline Grupo etáreo & Incidencia: Tasa $/ \mathbf{1 0 0 . 0 0 0}$ \\
\hline$<15$ años & 1,7 \\
15 - 64 años & 16,8 \\
$\geq 65$ años & 38,0 \\
\hline
\end{tabular}

Tabla 4.3. Número de baciloscopías de pesquisa. Región Metropolitana. 2001-2006

\begin{tabular}{lcccccc}
\hline Año & $\mathbf{2 0 0 1}$ & $\mathbf{2 0 0 2}$ & $\mathbf{2 0 0 3}$ & $\mathbf{2 0 0 4}$ & $\mathbf{2 0 0 5}$ & $\mathbf{2 0 0 6}$ \\
\hline Número de baciloscopias & 123.574 & 120.498 & 124.622 & 126.134 & 117.293 & 116.086 \\
\hline
\end{tabular}


Tabla 5.3. Casos de tuberculosis en grupos vulnerables. Región Metropolitana. 2006

\begin{tabular}{lcccc}
\hline Grupo vulnerable & \multicolumn{2}{c}{$\begin{array}{c}\text { Tuberculosis en todas las formas } \\
\text { Número de casos }\end{array}$} & \multicolumn{2}{c}{$\begin{array}{c}\text { Tuberculosis pulmonar bacilífera } \\
\text { Número de casos }\end{array}$} \\
\hline VIH & 56 & 5,7 & 25 & 5,5 \\
Inmigrantes & 35 & 3,6 & 14 & 3,1 \\
Reclusos & 31 & 3,2 & 18 & 4,0 \\
Indigentes & 12 & 1,2 & 8 & 1,8 \\
Adultos mayores & 197 & 20,0 & 68 & 15,0 \\
Total & 331 & 33,6 & 133 & 29,4 \\
\hline
\end{tabular}

* \%: porcentaje del total de casos

Tabla 6.3. Cohorte de 387 casos de tuberculosis bacilífera vírgenes a tratamiento ingresados a los Servicios de Salud, Región Metropolitana, 2005.

\begin{tabular}{lcr}
\hline Situación & Número de casos & $\%$ \\
\hline Alta (inactivos) & 312 & 80,6 \\
Abandonos & 31 & 8,0 \\
Fallecidos & 34 & 8,8 \\
Traslados & 10 & 2,6 \\
Fracasos & 0 & 0 \\
Total de ingresados & 387 & 100 \\
\hline
\end{tabular}

sos diagnosticados ya se encontraban en control médico en los servicios de salud por otras patologías, un $29 \%$ en control por diabetes y un $21 \%$ por EPOC.

Los resultados de la cohorte de 387 casos de tuberculosis pulmonar bacilífera vírgenes a tratamiento (VT), correspondiente a 2005 (Tabla 6.3), mostraron $80,6 \%$ de curaciones, con $8 \%$ de abandonos, $8,8 \%$ de fallecidos y un 2,6\% de traslados. No se presentaron fracasos. El seguimiento posterior de los pacientes pudo establecer que el $84,8 \%$ logró terminar el tratamiento, siendo dado de alta como inactivo, en tanto que los abandonos se redujeron a un 5,8\%; sin embargo, el porcentaje de fallecidos se mantuvo elevado $(8,1 \%)$.

\section{Proyectos para alcanzar la meta de eliminación de la tuberculosis}

Dr. Carlos Peña M. Coordinador regional metropolitano del programas de tuberculosis

Los Servicios de Salud de la Región Metropolitanos son una importante fuente de casos de tuberculosis $\mathrm{y}$, por lo tanto, gravitan en forma significativa en las tasas nacionales de tuberculosis. El retardo en la reducción de la incidencia puede comprometer la meta nacional de llegar al umbral avanzado de eliminación de la enfermedad como problema de salud pública el año 2010 (tasa < 10/100.000). Los servicios de la región metropolitana que deben apresurar el paso en la reducción de su problema epidemiológico son el SSM Occidente, el SSM Centro, el SSM Norte y el SSM Sur. Otros servicios del país, aunque tengan una peor situación epidemiológica, dada su reducida población, no influyen tanto en la incidencia nacional.

Para los servicios actualmente retrasados en el cumplimiento de las metas del MINSAL resulta esencial incrementar la pesquisa, con una focalización adecuada, a fin de detectar más precozmente las fuentes comunitarias de transmisión, y, conjuntamente, optimizar los resultados del tratamiento.

Para cumplir estos objetivos, se considera necesaria una planificación estratégica, con actividades especiales en los siguientes aspectos:

1. Actividades de detección activa de casos de tuberculosis transmisible;

2. Aumento de la eficiencia terapéutica en los casos bacilíferos, y

3. Optimización en la prevención de la tuberculosis en poblaciones de riesgo.

\section{Actividades de detección activa de casos}

a) En recintos penales en los sintomáticos respiratorios y mediante un examen de ingreso en los reclusos nuevos. Incluir en el estudio de contactos las visitas y familiares de los presos, así como los compañeros cercanos.

b) En pacientes con patologías crónicas bajo control en los consultorios de Atención Primaria, especialmente diabéticos, EPOC (salas ERA) y en adultos mayores. 
c) En consultantes en Servicios de Urgencia, a través de su personal de colaboración médica, para lo cual se debe mantener un suministro adecuado de contenedores para las muestras de expectoración, un transporte expedito al laboratorio y formularios simplificados.

\section{Aumentar eficiencia del tratamiento}

2.1. Estrategias de prevención del abandono, mediante la detección precoz del paciente en riesgo de abandono y aplicación de las medidas conducentes a reducir este riesgo. Un factor a considerar siempre es el efecto positivo antiabandono que tiene un DOTS bien llevado, lo que determina una sensación de seguridad para el enfermo.

Las medidas más importantes para evitar el abandono son:

2.1.1. En pacientes con dificultades para asistir al tratamiento supervisado, proporcionar los medios de transporte o los recursos económicos necesarios para subvencionar su movilización. En los casos de imposibilidad de traslado (postrados, etc), administrar la terapia en el domicilio del enfermo con personal capacitado.

2.1.2. En enfermos poco cooperadores, entrevista inicial, mayor educación y visita de asistente social al domicilio, involucrando al resto de la familia en el tratamiento. Si hay problemas laborales, reinsertarlo precozmente a sus actividades al momento de comprobar que no existe riesgo de contagio y trasladar la terapia a consultorios cercanos a su trabajo. En algunos casos puede ser necesaria una evaluación del estado mental del paciente, para tratar eventuales alteraciones asociadas (depresión, psicosis, etc).

2.1.3. En los enfermos en mala condición socioeconómica, desnutridos o con intolerancia digestiva menor, suministrarles antes de la terapia un alimento (jugo, leche, etc).

2.1.4. En los pacientes con reacciones adversas a drogas, manejar el problema en forma precoz, evitando el abandono producto de estas reacciones.

2.2. Estrategias de prevención de mortalidad tanto por tuberculosis como por patologías asociadas. Entre las medidas más importantes tenemos:

2.2.1. Hospitalización oportuna en centros hospitalarios, sanatorio o casas de colocación de los servicios de salud. Siempre se debe verificar si se requiere de algún grado de aislamiento, para seleccionar la sala apropiada.
2.2.2. Control de patologías asociadas, como diabetes, EPOC, etc. asegurando una rápida recepción del caso en las especialidades requeridas.

2.2.3. Detección y terapia precoz de las reacciones adversas severas con riesgo potencial de muerte, como la toxicidad hepática, trombocitopenia y anafilaxia.

2.2.3 Suprimir el consumo de alcohol y manejar las interacciones de fármacos.

\section{Prevención de la tuberculosis en poblacio- nes de riesgo}

Las situaciones más frecuentes a considerar son:

3.1. Quimioprofilaxis en VIH/SIDA según las normas del programa de Control de la Tuberculosis (PCT).

3.2. Estudio ampliado de contactos de casos bacilíferos. Puede ser aconsejable el seguimiento de los contactos a los 3, 6 y 12 meses, con repetición del estudio radiológico y baciloscopía. El estudio de contactos es de alta prioridad; debe ser completado independientemente de si el caso índice ya se encuentra de alta y debe incluir visitas domiciliarias seriadas y entrevistas sociales. Los exámenes requeridos deben ser facilitados por la gestión del programa, como por ejemplo, utilizando los cupos radiológicos.

3.3. El aislamiento inicial de los casos hospitalizados sospechosos de tuberculosis sin conocimiento de su estado bacteriológico.

3.4. Reducción del riesgo de exposición en situaciones, condiciones o lugares físicos de mayor riesgo para el personal de salud: laboratorio microbiológico, funcionarios de policlínico y recintos donde se efectúan autopsias y broncoscopías. En las normas del PCT hay un capítulo de bioseguridad que debe tenerse presente.

3.5. Participación en actividades de extensión, fuera de los consultorios y hospitales, como la instalación de pesquisa baciloscópica y centros de tratamiento en hospederías, Hogar de Cristo y Comunidades de inmigrantes, para lo cual se requiere de la formación de líderes capacitados o monitores de salud. Una alternativa promisoria la constituye la reconocida labor de los voluntarios de "Chile solidario", que pueden interactuar con los pacientes "en condición de calle", para la pesquisa y supervisión del tratamiento. Estos voluntarios deben ser capacitados dentro de las actividades del PCT de los servicios de salud. 